\title{
Cardiovascular Risk, Obesity, and Sociodemographic Indicators in a Brazilian Population
}

\author{
Nayla Cristina do Vale Moreira ${ }^{1,2,3 *}$, Ibrahimu Mdala ${ }^{4}$, Akhtar Hussain $^{2,3,5,6}$, \\ Bishwajit Bhowmik ${ }^{5}$, Tasnima Siddiquee ${ }^{5}$, Virgínia Oliveira Fernandes ${ }^{2}$, \\ Renan M. Montenegro Jr. ${ }^{2}$ and Haakon E. Meyer ${ }^{1,7}$
}

${ }^{1}$ Department of Community Medicine and Global Health, Institute of Health and Society, University of Os/o (UiO), Os/o, Norway, ${ }^{2}$ Faculty of Medicine, Federal University of Ceará (FAMED-UFC), Fortaleza, Brazil, ${ }^{3}$ Faculty of Health Sciences, NORD University, Bodø, Norway, ${ }^{4}$ Department of General Practice, Institute of Health and Society, University of Oslo (UiO), Oslo, Norway, ${ }^{5}$ Centre for Global Health Research, Diabetic Association of Bangladesh, Dhaka, Bangladesh, ${ }^{6}$ International Diabetes Federation, Brussels, Belgium, ${ }^{7}$ Norwegian Institute of Public Health, Oslo, Norway

OPEN ACCESS

Edited by:

Su Yon Jung,

University of California, Los Angeles,

United States

Reviewed by:

Xiangzhu Zhu,

Vanderbilt University, United States

Prof Asher Fawwad,

Baqai Medical University, Pakistan

*Correspondence:

Nayla Cristina do Vale Moreira

naylacristinam@yahoo.com.br

Specialty section:

This article was submitted to Life-Course Epidemiology and Social

Inequalities in Health,

a section of the journal

Frontiers in Public Health

Received: 14 June 2021 Accepted: 26 October 2021

Published: 30 November 2021

Citation:

Moreira NCdV, Mdala I, Hussain A

Bhowmik B, Siddiquee T,

Fernandes VO, Montenegro RM Jr and Meyer HE (2021) Cardiovascular Risk, Obesity, and Sociodemographic Indicators in a Brazilian Population.

Front. Public Health 9:725009.

doi: 10.3389/fpubh.2021.725009
Background and Aims: Cardiovascular diseases (CVDs) are the leading cause of death globally and in Brazil. Evidence suggests that the risk of CVDs differs by race/ethnicity. Scarce information exists about the association between CVD risk, obesity indicators and sociodemographic characteristics in the Brazilian population.

Objectives: We aimed to assess the CVD risk following the Framingham risk score in relation to the population's sociodemographic profile. Further, we examined the association between anthropometric markers and risk of CVDs.

Methods: A total of 701 subjects aged $\geq 20$ years from North-eastern Brazil were recruited randomly to participate in a population-based, cross-sectional survey. Age-adjusted data for CVD risk, sociodemographic characteristics, and anthropometric indices were assessed, and their relationships examined.

Results: High CVD risk (Framingham risk score $\geq 10 \%$ ) was observed in $18.9 \%$ of the population. Males (31.9 vs. $12.5 \%$ ) and older subjects (age $\geq 45$ years: $68.9 \%$ vs. age < 45 years: $4.2 \%$ ) had significantly higher risk of CVDs, whereas those employed in manual labor showed lower risk (7.6 vs. $21.7 \%$ ). Central obesity measures like waist-to-hip ratio and waist-to-height ratio were more strongly associated with predicted CVD risk than body mass index.

Conclusions: Our population had a high risk of CVDs using the Framingham risk score. Cost-effective strategies for screening, prevention and treatment of CVDs may likely reduce disease burden and health expenditure in Brazil. Central obesity measures were strongly associated with predicted CVD risk and might be useful in the clinical assessment of patients. Follow-up studies are warranted to validate our findings.

Keywords: cardiovascular risk (CVD), Framingham risk score (FRS), obesity, sociodemographic indicators, anthropometric markers 


\section{INTRODUCTION}

Cardiovascular diseases (CVDs) have reached epidemic proportions worldwide, with a greater impact in low- and middle-income countries (LMICs), including Brazil (1). In 2016, approximately 17.9 million people died from CVDs globally, mostly due to heart attack and stroke. Over $75 \%$ of these deaths have taken place in LMICs (2). CVDs are the leading cause of death in Brazil and responsible for the highest healthcare expenditure for hospital admissions (3).

Most CVDs are caused by a complex interaction of several modifiable risk factors, including tobacco use, physical inactivity, unhealthy diet, overweight and obesity, harmful use of alcohol, hypertension, diabetes, and dyslipidaemia (1). Recently, Brazil has experienced a rapid demographic and economic transition, resulting in profound changes in nutritional and lifestyle patterns. Industrialization, urbanization, an aging population, and increased prevalence of unhealthy habits have become root causes of the rising CVD burden in Brazil (3). Amongst these risk factors, obesity is an increasing concern. According to 2016 estimates, around $22 \%$ of Brazilian adults aged $\geq$ 18 years and $9 \%$ of adolescents aged 10-19 years were obese (4).

Overweight and obesity have been regarded as one of the leading factors for mortality, accounting for around $23 \%$ of the ischaemic heart disease burden $(5,6)$. Several anthropometric measures of general and central obesity have been applied to assess adiposity-related risk, including body mass index (BMI), waist circumference (WC), waist-to-hip ratio (WHR), and waist-to-height ratio (WHtR) (5). However, previous studies have found conflicting results regarding the usefulness of these different anthropometric indices (7-9). Moreover, even though most of the global burden of CVDs is in developing countries, the existing evidence is derived mainly from high-income countries (10). Since adiposity is highly heterogeneous with age, gender, and ethnicity (11), it remains unclear which anthropometric parameters are better correlated with the risk of CVDs in different populations (12).

Evidence suggests that the risk of CVDs differs by race/ethnicity (13). In Brazil, although several studies were conducted for the prevalence of cardiovascular risk factors, most of them have limitations due to potential selection bias and the use of self-reported data in the absence of confirmatory laboratory examinations (14). Moreover, few studies have compared the independent associations between the different anthropometric indices and CVD risk based on the recommended cut-off values (15). Scarce information exists in Brazil about the risk of CVDs and sociodemographic and anthropometric characteristics of the population. Thus, in this cross-sectional, population-based study, we aimed to investigate CVD risk following the Framingham risk score and how it is related to socioeconomic and demographic characteristics. We also studied the association between some anthropometric markers, i.e., WC, BMI, WHR and WHtR, and the predicted risk of CVDs in both genders.

\section{MATERIALS AND METHODS}

\section{Study Population}

This cross-sectional study was carried out between August 2012 and January 2013 in the city of Pindoretama, located in the state of Ceara (CE), North-eastern Brazil. The recruitment and examination procedures have been discussed previously (16). According to the latest demographic census conducted in 2010, the total population of Pindoretama was approximately 18,683 inhabitants (17). The health registry list with the citizens' names in alphabetic order was applied to select the potential study subjects. Random numbers were generated with the statistical software $\mathrm{R}$ (18) and identified with the names in the list thereafter. The selected subjects were invited to participate in the survey by local Community Health Workers (CHW). Around 1,000 subjects were randomly selected based on the list. Of these, one hundred and sixty-three were not found by the CHW and, therefore, could not receive the invitation. Thirty-one subjects did not meet the inclusion criteria. Thus, eight hundred and six randomly selected subjects were invited, of whom 714 agreed to participate (a response rate of $88.6 \%$ ).

Subjects of both genders, aged $\geq 20$ years who were able to communicate and willing to participate in the study were considered eligible. Those with an acute or chronic severe cardiac, renal, or hepatic illness, as well as physically or mentally disabled subjects unable to follow simple questions and examinations were excluded, as were pregnant women. Since we aimed to assess the CVD risk, those with a previous history of myocardial infarction and/or stroke were considered as having had the condition, and therefore were excluded from the analyses (13 subjects). Seven hundred and one subjects remained. At the time of recruitment, the subjects were requested to visit a nearby health center after an overnight fast of 8-10 h. Pre-tested questionnaires were conducted by trained interviewers to collect sociodemographic and clinical information. Anthropometric measurements, blood pressure, and body fat percentage (BF\%) were also registered.

\section{Sample Size Calculation}

The required sample size was calculated by the formula: $n=4$ $\left(z_{\text {crit }}\right)^{2} p(1-p) / D^{2}(19)$. The total sample size was represented by " $n$ ", " $z_{\text {crit }}$ " $=1.96$ (Standard Normal Deviate for a Significance Criterion $=0.05$ and a Confidence Interval $=0.95$ ), “ $p "=0.051$ prevalence estimate from a previous study of high/intermediate risk of CVD according to the Framingham risk score (20), and " $D$ " $=0.0454$ (total width of the expected confidence interval). Two-tailed statistical analyses were used. Thus, $n=4 \times(1.96)^{2}$ $\times 0.051 \times 0.949 /(0.0454)^{2} ; n=360.83$.

\section{Ethics}

The study was conducted according to the ethical principles outlined in the Helsinki Declaration (21). The research protocol was approved by the local Ethical Committee in Brazil (Protocol Number: 045.06.12) and the Regional Committee for Medical and Health Research Ethics (REK) in Norway (Reference: 2012/779/REK sør-øst D). Written or verbal consent was sought from each subject prior to any investigation. The subjects were 
informed of their right to withdraw from the study at any point or withhold their data from the analysis. Those who were diagnosed with any clinical condition were referred to the nearest health center for treatment and follow-up.

\section{Measurements}

Weight, height, WC, and hip circumference (HC) were taken with subjects standing without shoes and wearing light clothing. Body weight (kilograms) was registered to the nearest $0.1 \mathrm{~kg}$ using a portable digital scale, calibrated before use and checked every day with a known weight. Height (centimeters) was measured by applying a well-mounted stadiometer, with each subject standing upright with their head in the Frankfurt plane. BMI was calculated as the weight in kilograms divided by the square of the height in meters $\left(\mathrm{kg} / \mathrm{m}^{2}\right) . \mathrm{BF} \%$ was determined by a portable bipolar body fat analyser (Omron $\AA$, Model HBF306, Omron Healthcare, Inc., Illinois, United States). WC was measured with a non-stretchable tape, positioned horizontally in the middle area between the lower border of the ribs and iliac crest, under the mid-axillary line. HC was assessed with the same tape positioned to the maximum circumference around the buttocks, with the subjects standing straight. WC and HC were recorded to the nearest $0.1 \mathrm{~cm}$. WHR was calculated as the WC divided by the HC, while the WHtR as the WC divided by the height.

Blood pressure $(\mathrm{mmHg})$ was estimated twice at a 10min interval using a validated automatic sphygmomanometer (Omron $\AA$ BP785 IntelliSense $\AA$ Automatic Blood Pressure Monitor with ComFitTM Cuff, Omron Healthcare, Inc., Illinois, United States), with appropriate cuffs, in a sitting position after a resting time of at least $15 \mathrm{~min}$. The mean of the two readings was used for the analysis.

\section{Blood Sampling and Laboratory Assays}

On arrival at the data collection center, a $10-\mathrm{mL}$ fasting venous blood sample was taken to determine the concentrations of plasma glucose, insulin, and lipids. Two hours after a $75 \mathrm{~g}$ oral glucose load, another $3 \mathrm{ml}$ of venous blood was drawn for the oral glucose tolerance test (OGTT). Blood samples were stored immediately over ice and centrifuged after $1 \mathrm{~h}$. Plasma was frozen and transported to the laboratory, where the samples were stored at $-20{ }^{\circ} \mathrm{C}$ until the analyses were conducted. Quality control of the laboratory was assessed internally and externally.

The glucose oxidase method was applied to estimate fasting and 2-h plasma glucose levels, whereas fasting insulin was determined by chemiluminescence. Total cholesterol (TC) was assessed by the cholesterol oxidase - phenol + aminophenazone (CHOD-PAP) method, while a homogenous enzymatic colorimetric method was used to determine highdensity lipoprotein cholesterol (HDL-C) levels. Triglycerides (TG) were assessed by the glycerol-3-phosphate oxidase phenol + aminophenazone (GPO-PAP) method. The Friedewald Formula (22) was applied to calculate the low-density lipoprotein cholesterol (LDL-C) levels.

\section{Definitions of Variables}

The cut-off points for WHR recommended by the World Health Organization (WHO) were applied, i.e., for males, a WHR $\geq$ 0.90 was classified as "high" (substantially increased risk of metabolic complications), whereas, for females, a WHR $\geq 0.85$ was considered "high". Overweight/obese was defined by a BMI of $\geq 25 \mathrm{~kg} / \mathrm{m}^{2}$. A high WC was described as $>102 \mathrm{~cm}$ for males, and $>88 \mathrm{~cm}$ for females (23). A cut-off of $\geq 0.50$ was applied to define a high WHtR (24).

Following the definition of the Brazilian Institute of Geography and Statistics (IBGE) classification, ethnicity was assessed according to the subjects' self-perception of their skin color. The different ethnic groups were categorized into "white" and "non-white" (17). Physical activity information was ascertained by the International Physical Activity Questionnaire (IPAQ) short form (25). The IPAQ's total score was computed by summing up the duration and frequency of walking, moderateand vigorous-intensity activities. Following the guidelines for data processing and analysis, the levels of physical activity were classified into "low", "moderate" and "high" (26). We further categorized them into "low" and "moderate" plus "high" level. Current smoking included those who self-reported as being smokers or had stopped smoking for less than 1 year. Alcohol consumption was ascertained by self-report as yes/no. The occupation of the subjects was categorized into manual and non-manual labor. Manual labor was used to describe jobs in agriculture and construction, whereas non-manual labor described all other occupations.

The 1999 WHO criteria (27) were applied in diagnosing diabetes mellitus. Diabetes cases were defined as those who had a previous diagnosis of type 2 diabetes, or those with fasting (venous) plasma glucose value $\geq 7.0 \mathrm{mmol} / \mathrm{l}$ ( $\geq 126 \mathrm{mg} / \mathrm{dl}$ ), or the 2 -h plasma glucose value after a $75 \mathrm{~g}$ oral glucose load $\geq 11.1$ $\mathrm{mmol} / \mathrm{l}(\geq 200 \mathrm{mg} / \mathrm{dl})$, or both. Dyslipidaemia was defined as $\mathrm{TG} \geq 1.7 \mathrm{mmol} / \mathrm{l}$ and $\mathrm{HDL}<0.9 \mathrm{mmol} / \mathrm{l}$ for males; and $<1.0$ $\mathrm{mmol} / \mathrm{l}$ for females (27). Insulin resistance was estimated by the homeostasis model assessment of insulin resistance (HOMA-IR $=[$ insulin $(\mathrm{mU} / \mathrm{l}) \times$ glucose $(\mathrm{mmol} / \mathrm{l})] / 22.5)(28)$. Hypertension was defined as systolic blood pressure (SBP) $\geq 140 \mathrm{mmHg}$ and/or diastolic blood pressure (DBP) $\geq 90 \mathrm{mmHg}$ and/or being on blood-pressure-lowering medication (29).

\section{Estimating the Framingham Risk Score}

The Framingham 10-year risk score model, as published by D'Agostino et al. (30) in 2008, was applied to estimate the predicted 10-year risk for an incident cardiovascular event. The model predictors included age, gender, SBP, use of antihypertensive medication, TC, HDL-C, smoking and diabetes status (30). Subjects with a Framingham predicted risk of $10 \%$ or above during the next 10 years were defined as having high CVD risk. Although data were collected from 701 subjects in total, the Framingham risk score was estimated for 693 subjects owing to missing values (229 males and 464 females). In Figures 1, 2, based on D'Agostino et al. (30) and Chang et al. (31), we presented the mean Framingham risk score estimates for the age range 30-74 years. 


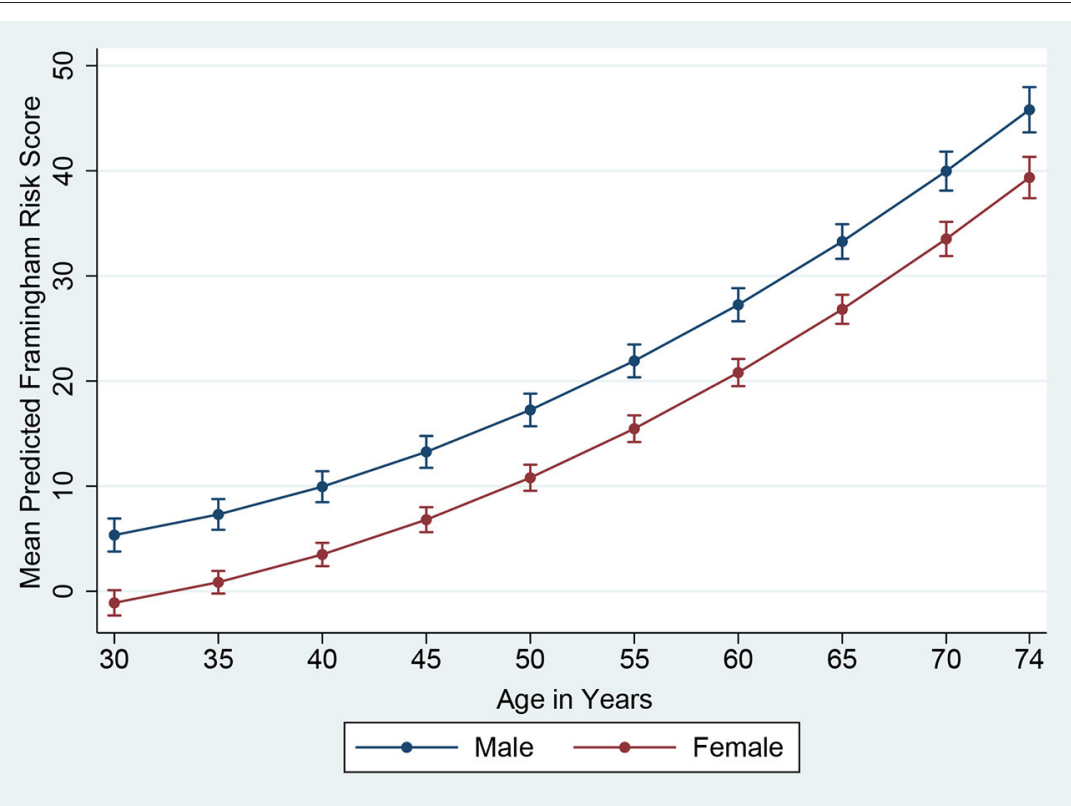

FIGURE 1 | Framingham Risk Score by age and gender (vertical lines are means with 95\% Cls).

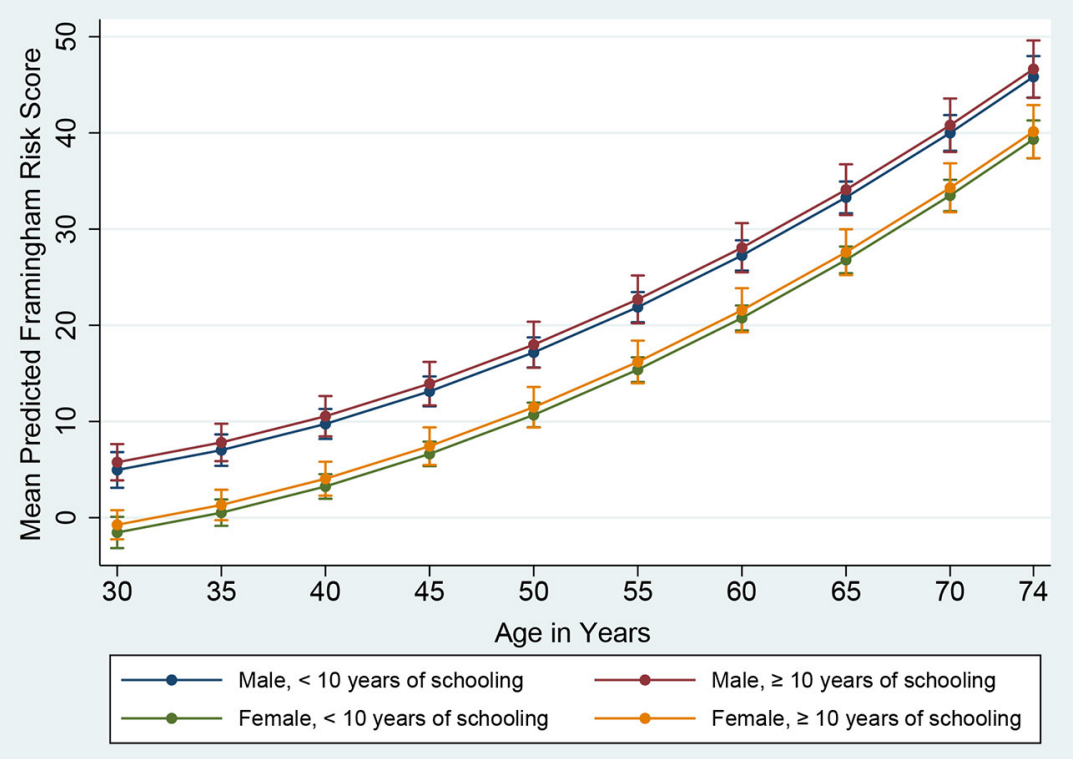

FIGURE 2 | Framingham Risk Score by age, gender, and education (vertical lines are means with 95\% Cls).

\section{Statistical Analysis}

Continuous data were expressed as means and $95 \%$ confidence intervals (CIs), while percentages and 95\% CIs were given for categorical variables. Generalized linear regression models (GLM) were fitted to the data after adjusting for age. To compare differences between adjusted means, we fitted GLMs with linear link function, while GLMs with the logit link function were applied to compare differences between proportions. The prevalence of those with a predicted 10-year CVD risk of $\geq 10 \%$ was calculated as predictive margins, based on the estimation of the adjusted logistic regression models. To control confounding by age in the predicted means and prevalence, we fixed age at 45 years, which was the closest to the mean age in the sample. A two-sample test of proportions was applied to compare the 
prevalence of high CVD among the different sociodemographic groups. Anthropometric measurements were converted to zscores [original value subtracted by the mean and divided by the standard deviation (SD)] to represent the number of SDs above and below the mean for each subject. Multiple linear regression was carried out to investigate the relationship between the standardized anthropometric markers and CVD risk. Further, we calculated crude and adjusted prevalence ratios (PRs) of the anthropometric indicators for detecting high CVD risk using Poisson regression with robust variance, as the prevalence of high CVD risk was above $10 \%$. We tested for two-way interaction between age and the different anthropometric markers. The Akaike Information Criteria (AIC) was used to compare nested models. Data were analyzed using Stata $15^{\text {th }}$ edition (32) and SPSS $26^{\text {th }}$ version (33) statistical software. The results were considered statistically significant with $p<0.05$, and all tests were two-sided.

\section{RESULTS}

A total of 701 subjects (234 males and 467 females, mean age 44.8 $\pm 16.0 \mathrm{SD}$ ) were included in the analysis. Significant differences were found in sociodemographic, lifestyle, anthropometric and cardiometabolic characteristics between the genders (Table 1). Males had a significantly higher proportion of tobacco smoking and alcohol consumption. Females were more physically inactive and showed a higher percentage of overweight/obesity. Age, dyslipidaemia, hypertension, and diabetes status did not differ between the genders. Anthropometric parameters including mean HC, WHtR, BMI and BF\% were higher in females, while males had a higher WHR.

The mean predicted Framingham risk score increased substantially with age and was higher among males (Figure 1). In addition, the mean predicted risk was not statistically significant between different levels of education in both genders (Figure 2).

As shown in Table 2, the estimated proportion with a predicted 10 -year CVD risk of $\geq 10 \%$ was significantly higher among males ( 31.9 vs. $12.5 \%$; $p$-value: $<0.001)$, and those with more than 45 years of age $(68.9$ vs. $4.2 \%$; $p$-value: $<0.001)$. Furthermore, it was significantly lower among those with an occupation requiring manual labor (7.6 vs. $21.7 \%$; $p$-value: 0.008 ), defined as jobs in agriculture and construction.

Multiple linear regression was carried out to assess the age-adjusted associations between $1 \mathrm{SD}$ increment in each anthropometric marker and the predict risk of CVDs, entered as a continuous variable (Table 3). In males, only WHtR was a significant predictor of CVD risk followed by a borderlinesignificant association for WC, while in females WHR and WHtR were statistically significant. WHtR showed the highest slope coefficient in males, whereas WHR had the highest slope in females.

Table 4 presents the PRs of the different anthropometric measures for identifying a high CVD risk. Univariable and multivariable Poisson regression analyses with robust variance were applied. Adjusted PRs were obtained after controlling for age, level of physical activity, family history of cardiac disease and stroke. An interaction term between age and the corresponding anthropometric parameter was included in some adjusted models, according to their statistical significance and the Akaike Information Criteria (AIC). In females, significant positive associations were found between all anthropometric variables and high CVD risk in the adjusted models, except for WC. In males, all anthropometric markers were significant. WHtR had the highest adjusted PR for males (9.9, 95\% CI: $2.8-$ $34.8, p$-value $<0.001)$ and females (43.4, 95\% CI: 2.6-716.8, $p$ value 0.002 ). This large $\mathrm{PR}$ and wide $\mathrm{CI}$ in the adjusted model for females and WHtR was due to the few observations of those with high CVD risk and WHtR $<0.50$ (6 subjects).

\section{DISCUSSION}

To the best of our knowledge, this is one of the few populationbased studies from Brazil to investigate the CVD risk by sociodemographic characteristics, as well as the association between different obesity markers and the risk of a cardiovascular event. Males and older people presented higher risk of CVDs in our population, whereas those employed in the manual labor had significantly lower risk. Central obesity measures were more strongly associated with CVD risk than BMI.

We found a high prevalence of increased CVD risk, i.e., Framingham risk score $\geq 10 \%$, in this population. Our estimates were higher than those reported in Peru (34), Argentina (35) and Southern Brazil $(20,36)$, similar to India (37), but lower than Honduras (38) and China (39). These differences might be explained by genetic, racial, sociodemographic, and cultural diversity, as well as the use of other versions of the Framingham risk score, with a varied set of predictors. In our sample, the prevalence of diabetes, smoking, hypertension, and dyslipidaemia was higher than reported in some other Brazilian surveys (3). The recent rapid industrialization and urbanization of Pindoretama (the rural population decreased from $66 \%$ to 39\% between 1991 and 2010) (40), resulting in lifestyle and dietary changes, might explain the frequent occurrence of these cardiovascular risk factors and subsequent high Framingham risk score in the studied population.

Consistent with previous research $(35,37,41,42)$, males had a higher Framingham risk score than females. This might be due to the significantly higher SBP, and tobacco use among males. As expected, the Framingham risk score increased significantly with age, which is also in line with other studies $(36,41)$. The subjects employed in in agriculture or construction showed lower CVD risk, possibly reflecting the protective effect of physical activity (1). After controlling for age and gender, among those employed in manual labor, about $60.4 \%$ presented a moderate to high level of physical activity, whereas only $30.5 \%$ of those in other employment categories were similarly active (data not shown). On the other hand, the CVD risk did not differ significantly among the ethnic groups. This might be explained by the mixed genetic composition of the Brazilian population, essentially formed by an admixture of native Brazilians, Europeans, and Africans (43). It is likely that the extensive miscegenation of the overall Brazilian population may have reduced the differences among the ethnic groups. Although other studies have found an inverse relationship between CVD risk and education, our data did not find significant results. The relationship between education and risk of CVDs has shown great variability across 
TABLE 1 | Baseline characteristics of the study subjects.

\begin{tabular}{|c|c|c|c|c|}
\hline Characteristics & All $(n=701)$ & $\operatorname{Males}(n=234)$ & Females $(n=467)$ & $p$-value* \\
\hline Age (years) & $44.8(43.6-46.0)$ & $45.6(43.6-47.7)$ & $44.4(42.9-45.8)$ & 0.319 \\
\hline \multicolumn{5}{|l|}{ Ethnicity (\%) } \\
\hline White & $16.6 \%$ (13.8-19.3) & $10.7 \%(6.7-14.6)$ & $19.5 \%(15.9-23.1)$ & 0.003 \\
\hline Non-white & $83.4 \%(80.7-86.2)$ & 89.3\% (85.4-93.3) & $80.5 \%(76.9-84.1)$ & \\
\hline \multicolumn{5}{|l|}{ Education (\%) } \\
\hline \multicolumn{5}{|l|}{ Monthly Income (\%) } \\
\hline$<2 \mathrm{MW}$ & $90.2(88.0-92.4)$ & $80.6(75.5-85.7)$ & 95.0 (93.0-96.9) & $<0.001$ \\
\hline$\geq 2 \mathrm{MW}$ & $9.8(7.6-12.0)$ & $19.4(14.3-24.5)$ & $5.0(3.1-7.0)$ & \\
\hline Manual Labor (\%) ** & $9.5(7.5-11.4)$ & $27.5(21.7-33.3)$ & $0.4(-0.2-1.0)$ & $<0.001$ \\
\hline Currently Married (\%) & $66.7(63.3-70.2)$ & $74.3(68.8-79.9)$ & $62.9(58.5-67.3)$ & 0.003 \\
\hline Low & $66.8(63.4-70.3)$ & $55.4(49.0-61.8)$ & $72.6(68.5-76.6)$ & $<0.001$ \\
\hline Moderate/High & $33.2(29.7-36.6)$ & $44.6(38.2-51.0)$ & $27.4(23.4-31.5)$ & \\
\hline WC (cm) & $90.1(89.2-91.0)$ & $89.6(88.0-91.2)$ & 90.4 (89.3-91.5) & 0.415 \\
\hline $\mathrm{HC}(\mathrm{cm})$ & $98.6(97.9-99.4)$ & $95.7(94.4-97.0)$ & $100.1(99.2-101.0)$ & $<0.001$ \\
\hline WHR, mean & $0.92(0.91-0.92)$ & $0.94(0.93-0.95)$ & $0.90(0.89-0.91)$ & $<0.001$ \\
\hline WHtR, mean & $0.57(0.56-0.58)$ & $0.54(0.53-0.55)$ & $0.59(0.58-0.60)$ & $<0.001$ \\
\hline $\mathrm{BMI}\left(\mathrm{kg} / \mathrm{m}^{2}\right)$ & $26.9(26.5-27.3)$ & $25.9(25.3-26.6)$ & $27.4(26.9-27.8)$ & $<0.001$ \\
\hline Overweight/Obese (\%) & $61.9(58.4-65.5)$ & $53.4(47.0-59.8)$ & $66.2(61.9-70.5)$ & 0.001 \\
\hline BF\%, mean & $32.8(32.3-33.4)$ & $24.8(23.9-25.7)$ & $36.9(36.2-37.5)$ & $<0.001$ \\
\hline $\mathrm{SBP}(\mathrm{mmHg})$ & $127.6(126.2-129.0)$ & $132.7(130.3-135.1)$ & $125.1(123.4-126.8)$ & $<0.001$ \\
\hline $\mathrm{DBP}(\mathrm{mmHg})$ & $76.8(75.6-78.1)$ & 77.7 (75.5-79.9) & $76.4(74.8-77.9)$ & 0.326 \\
\hline Dyslipidaemia (\%) & $24.8(21.6-28.0)$ & $24.2(18.7-29.8)$ & $25.0(21.1-29.0)$ & 0.817 \\
\hline
\end{tabular}

Data are mean (95\% confidence intervals) or percentage ( $95 \%$ confidence intervals). Model was evaluated at age 45 years; ${ }^{*} p$-value for the difference between males and females. ${ }^{\star *}$ Manual Labor = refers to jobs in agriculture and construction; ${ }^{* \star}$ Included those who self-reported as being smokers or had stopped smoking for less than 1 year. BF\%, Body Fat Percentage; BMI, Body Mass Index; DBP, Diastolic Blood Pressure; HC, Hip Circumference; HDL-C, High-Density Lipoprotein Cholesterol; HOMA-IR, Homeostasis Model Assessment of Insulin Resistance; LDL-C, Low-Density Lipoprotein Cholesterol; MW, Minimum Wage in 2012; SBP, Systolic Blood Pressure; WC, Waist Circumference; WHtR, Waist-to-Height Ratio; WHR, Waist-to-Hip Ratio.

populations, depending particularly on the level of health transition and socio-economic development of the country (31, 44). In 2010, the illiteracy rate in Pindoretama among those aged 15 and older was approximately $22 \%$, whereas in Brazil it was $10 \%$ (40). In our data, only $4 \%$ of the subjects had a university degree or higher (data not shown). Therefore, it is likely that the lack of significant association between education and CVD risk might be due to the overall low level of education in our sample.

We found that the adjusted PR for WHR and WHtR were the highest among the anthropometric indices in relation to increased CVD risk. Further, the association between the WHR and Framingham risk score entered as a continuous variable was higher than that of WC, BMI and WHtR in females, whereas the slope coefficient of WHtR was the highest in males followed by WC. These results may indicate that these central obesity measures were more predictive of CVD risk than the general obesity measure like BMI. Therefore, in line with several others $(5,10)$, our findings suggest that BMI alone is insufficient to account for the association between CVD risk and obesity in this population. Over recent years, accumulating evidence has shown that abdominal obesity is more strongly associated with metabolic and cardiovascular problems than total adiposity $(10,45)$. Even within normal ranges of BMI, high visceral fat deposition remains an independent cardiovascular risk factor 
TABLE 2 | Predicted proportions of subjects with 10-year CVD risk of $\geq 10 \%$ using the Framingham Risk Score by sociodemographic characteristics.

\begin{tabular}{|c|c|c|c|}
\hline Characteristics & $\mathbf{n}$ & $\begin{array}{c}\text { Predicted } \\
\text { 10-year risk } \geq 10 \% \\
\% \text { (95\% Cls) }\end{array}$ & $p$-value \\
\hline Overall & $693^{\star}$ & 18.9 (14.3-23.6) & \\
\hline \multicolumn{4}{|l|}{ Gender } \\
\hline Male & 229 & $31.9(21.8-42.0)$ & $<0.001$ \\
\hline Female & 464 & $12.5(8.0-17.0)$ & \\
\hline \multicolumn{4}{|l|}{ Age groups } \\
\hline$<45$ years & 388 & $4.2(2.2-6.2)$ & $<0.001$ \\
\hline$\geq 45$ years & 305 & $68.9(63.8-74.0)$ & \\
\hline \multicolumn{4}{|l|}{ Ethnicity } \\
\hline White & 116 & 20.8 (9.4-32.2) & 0.58 \\
\hline Non-white & 577 & $18.6(13.7-23.5)$ & \\
\hline \multicolumn{4}{|l|}{ Education } \\
\hline$<10$ years & 489 & $19.2(14.1-24.4)$ & 0.60 \\
\hline$\geq 10$ years & 204 & $17.5(7.4-27.7)$ & \\
\hline \multicolumn{4}{|l|}{ Monthly income } \\
\hline$<2 \mathrm{MW}$ & 623 & $17.7(12.8-22.7)$ & 0.11 \\
\hline$\geq 2 \mathrm{MW}$ & 68 & 25.6 (11.4-39.9) & \\
\hline \multicolumn{4}{|l|}{ Occupation $^{\star \star}$} \\
\hline Non-manual labor & 629 & $21.7(16.4-27.1)$ & 0.008 \\
\hline Manual labor & 64 & $7.6(1.3-13.9)$ & \\
\hline
\end{tabular}

Data are percentage (95\% confidence intervals), adjusted for age (at age fixed to 45 years) and gender. *The study collected data from 701 subjects in total, but due to some missing values, the Framingham Risk Score was calculated for 693 subjects (229 males and 464 females). ${ }^{*}$ Manual Labor: jobs in agriculture and construction. Non-manual Labor: other occupations. Cls: Confidence Intervals. CVD: Cardiovascular Disease. MW: Minimum Wage in 2012.

(45). Although BMI is strongly correlated with gold standard body fat measures, it cannot distinguish between lean and fat mass and does not delineate body fat distribution patterns (46). Whilst BMI would not account for an increase in muscle or fatfree mass, this would be reflected in the central obesity measures (5). Accumulation of visceral fat is related to insulin resistance, increased systemic inflammation, accelerated progression of atherosclerosis and endothelial dysfunction, which contribute to CVD risk $(5,45)$. This might explain the stronger association between abdominal obesity measures and CVD risk reported in our study. Generally, measures of obesity are not included in the prediction of CVD risk (5). Considering our findings, it might be beneficial to incorporate central obesity indicators such as WHR and WHtR into the clinical assessment of CVD risk.

Some studies have identified WC as the most highly correlated marker with CVD risk factors compared with other central obesity measures and BMI in females (47). Nevertheless, another cross-sectional study from Brazil including 270 women also reported that WHR showed a greater performance than WC in discriminating high coronary risk (15). Although WHR is more difficult to measure than $\mathrm{WC}$, it has been considered a more specific surrogate for fat distribution, presents high precision and no bias over several ethnic groups (5). Our study showed a strong association between WHtR and CVD risk. Contrary to our results, a systematic review and meta-analysis reported
TABLE 3 | Association between 1 SD increase in anthropometric markers and CVD risk, using the Framingham Risk Score, age adjusted.

\begin{tabular}{lccc}
\hline Characteristics & $\begin{array}{c}\text { Slope coefficient }(\beta)(95 \% \\
\text { Cls) }\end{array}$ & $p$-value & R square
\end{tabular}

\section{Males}

$\mathrm{BMI}$ (per 1 SD)

WHR (per 1 SD)

$1.67(-0.01-3.35)$

0.05

0.6754

$1.56(-0.06-3.19)$

0.6750

WHtR (per 1 SD)

$1.30(-0.48-3.09)$

0.6728

Females

WC (per 1 SD)

BMI (per 1 SD)

WHR (per 1 SD)

$1.82(0.09-3.56)$

0.15

0.6760

WHtR (per 1 SD)

$0.78(-0.12-1.69)$

0.04

0.5811

$0.60(-0.27-1.48)$

0.09

0.5801

$1.13(0.14-2.11)$

0.5830

$0.95(0.01-1.89)$

0.03

0.5820

"p-value for each predictor in the regression model, controlling for age. BMI, Body Mass Index; Cis, Confidence Intervals; CVD, Cardiovascular Disease; SD, Standard Deviation; WC, Waist Circumference; WHR, Waist-to-Hip Ratio; WHtR, Waist-to-Height Ratio.

that WHtR had the weakest association with CVD risk factors, compared with BMI and other measures of central obesity (47). However, other studies showed an opposite scenario in which WHtR was the most highly correlated obesity marker with CVD risk (5). Compared to WC, studies in different populations have described WHtR as a more sensitive indicator, possibly due to the adjustment to different statures and negative correlation of height to some metabolic risk factors (48).

This study contributes to the limited body of evidence from Brazil on CVD risk and sociodemographic characteristics, as well as on the association between different obesity measures and the risk of CVDs. The subjects were randomly selected, and the participation rate was high. The survey was performed by trained personnel and pre-tested questionnaires were applied. To minimize the risk of misclassification errors due to poor recall, anthropometric parameters were carefully assessed, and no self-reported measures were used. Blood samples were collected, handled, and transported according to standard protocols. Quality control of the laboratory was assessed internally and externally.

Our study had some limitations. It was based on a crosssectional design and the 10-year CVD risk was calculated instead of using prospective CVD events. Nevertheless, the study generated valuable epidemiological data from Brazil regarding the association between CVD risk, obesity indicators and sociodemographic characteristics. Considering that Brazil is a large country with marked socioeconomic, ethnic, and regional disparities, our findings may not be representative for the whole nation. Caution should be taken when generalizing the results. However, since Brazilians have a mixed background, our sample might be a fair representation of the country's population. Furthermore, we had an overrepresentation of females (females 467 vs. males 234). As previously mentioned, out of 1,000 names randomly selected from the healthy registry list, around 163 subjects were not found by the CHW and therefore could not be invited to participate in the study. Out of these 163, approximately $78 \%$ were males. Additionally, among 92 subjects 
TABLE 4 | Crude and adjusted prevalence ratios (PRs) of anthropometric indices for identifying high CVD risk ( $\geq 10 \%$ using the Framingham Risk Score).

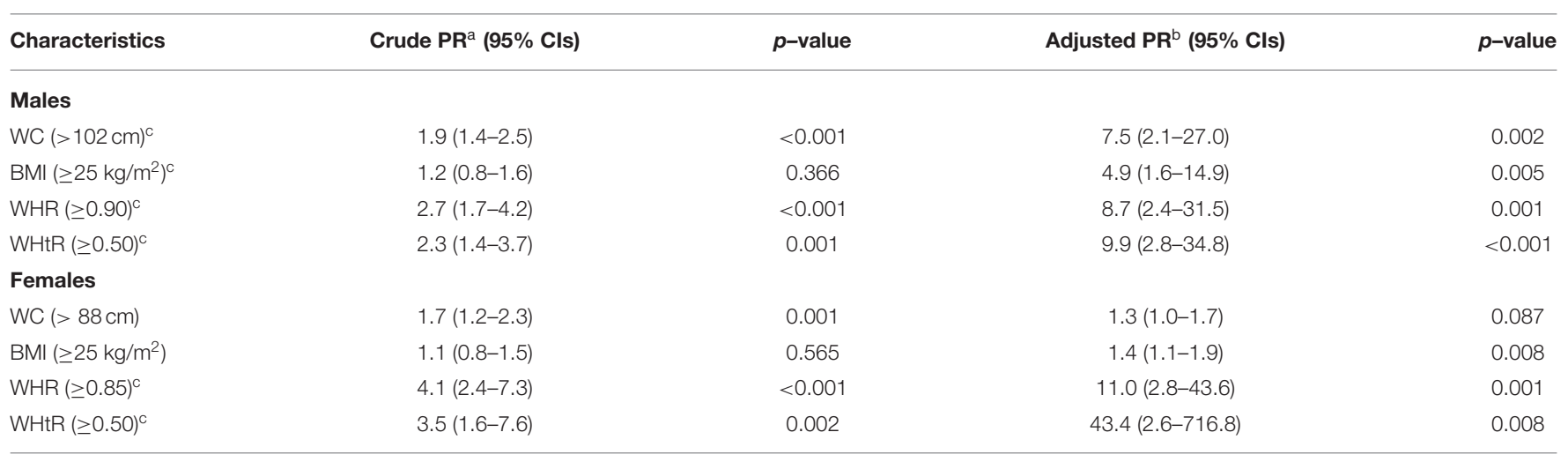

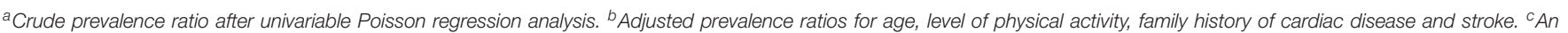

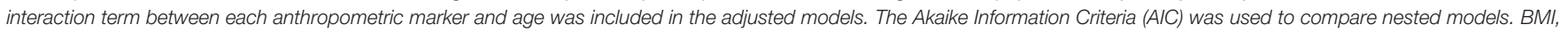
Body Mass Index; Cis, Confidence Intervals; CVD, Cardiovascular Disease; WC, Waist Circumference; WHR, Waist-to-Hip Ratio; WHtR, Waist-to-Height Ratio.

who refused to participate, around 63\% were males. Populationbased studies conducted during the day may constitute a hindrance to male participation. Males are often involved in income-generating work and therefore may not have been able to participate in the survey. The overrepresentation of females was dealt with by adjusting the analyses for gender or stratifying by gender. The Framingham risk score was not recalibrated for our population, which might have introduced some uncertainty in the CVD risk estimation. However, this was beyond the scope of the study and our available resources. Furthermore, the Framingham risk score has been widely applied and validated in ethnically diverse cohorts including whites, blacks, Native Americans, and Hispanics (49).

A high risk of CVDs according to the Framingham risk score was found in this population, especially among males and older people. In addition, manual labor seems to provide a protective effect on CVD risk. A timely targeted investment in screening, prevention, and necessary treatment of CVDs could reduce the burden on many and reduce the pressure on the health budget. Central obesity measures are more strongly associated with CVD risk than general obesity indicators. Our data suggest that WHR and WHtR are the best anthropometric markers to identify high CVD risk. Since an increase in muscle mass might not lead to changes in BMI (5), central obesity markers might be more useful to evaluate the effect of lifestyle changes in relation to CVD risk. Therefore, measuring WHR or WHtR might be beneficial in the clinical assessment of CVD risk. Prospective studies are still needed to further elucidate future risk of CVDs and their relationship with obesity in Brazil.

\section{DATA AVAILABILITY STATEMENT}

The raw data supporting the conclusions of this article will be made available by the authors, without undue reservation.

\section{ETHICS STATEMENT}

The studies involving human participants were reviewed and approved by the Local Ethical Committee in Brazil (Protocol
Number: 045.06.12) and the Regional Committee for Medical and Health Research Ethics (REK) in Norway (Reference: 2012/779/REK sør-øst D). The patients/participants provided their written informed consent to participate in this study. In case of illiteracy, verbal consent was assured by a local witness, who signed the informed consent, to secure the free participation of the subjects.

\section{AUTHOR CONTRIBUTIONS}

NM contributed to the conceptualisation of research goals and design, drafting of the article, as well as acquisition, analysis, and interpretation of the data. AH contributed to the study concept and design, data analyses, writing the initial draft and revising it critically, study oversight, and leadership. BB participated in the analysis and interpretation of data, writing the article, and revising it critically. IM took part in conceptualizing the study aims and design, writing the initial draft and revising it critically, as well as organizing the database, and conducting the statistical analysis. TS contributed to the design of the study, data curation and analyses, and drafting of the article. VF contributed to conceptualizing the work and designing the methodology, data collection and analyses, writing the initial draft, and revising it critically. RM contributed to the study concept and design, data analyses, study management and coordination, as well as writing the initial draft, and revising it critically. HM contributed to the design of the methodology, data analyses, study oversight and leadership, writing the initial draft, and performing critical review. All authors read and approved the content of the final manuscript. Furthermore, they agreed to be accountable for all aspects of the work in ensuring that questions related to the accuracy or integrity of any part of the work are appropriately investigated and resolved.

\section{FUNDING}

This study was funded by the Ivar Helles Foundation, and the Health Department of the city of Pindoretama-CE. 


\section{ACKNOWLEDGMENTS}

We acknowledge the valuable contribution of our survey team members, lab technicians and community health workers for their continuous effort in the data collection. We are grateful to all subjects in the study for their active

\section{REFERENCES}

1. World Health Organization. Global Atlas on cardiovascular disease prevention and control (2011) Available online at: https://www.who.int/ cardiovascular_diseases/publications/atlas_cvd/en/.

2. World Health Organization. Cardiovascular diseases (CVDs). Key facts (2017) Available online at: https://www.who.int/en/news-room/fact-sheets/detail/ cardiovascular-diseases-(cvds).

3. Schmidt MI, Duncan BB, e Silva GA, Menezes AM, Monteiro CA, Barreto SM, et al. Chronic non-communicable diseases in Brazil: burden and current challenges. The Lancet. (2011) 377:1949-61. doi: 10.1016/S0140-6736(11)60135-9

4. World Health Organization. Noncommunicable Diseases Country Profiles. Geneva (2018).

5. Goh LG, Dhaliwal SS, Welborn TA, Lee AH, Della PR. Anthropometric measurements of general and central obesity and the prediction of cardiovascular disease risk in women: a cross-sectional study. BMJ Open. (2014) 4:e004138. doi: 10.1136/bmjopen-2013-004138

6. World Health Organization. Obesity and Overweight. Key facts (2018) Available online at: https://www.who.int/en/news-room/fact-sheets/detail/ obesity-and-overweight.

7. Satoh H, Kishi R, Tsutsui H. Body mass index can similarly predict the presence of multiple cardiovascular risk factors in middle-aged Japanese subjects as waist circumference. Internal Med. (2010) 49:97782. doi: 10.2169/internalmedicine.49.3006

8. Ying X, Song ZY, Zhao CJ, Jiang Y. Body mass index, waist circumference, and cardiometabolic risk factors in young and middle-aged Chinese women. $J$ Zhejiang Univ Sci B. (2010) 11:639-46. doi: 10.1631/jzus.B1000105

9. Zhu S, Heymsfield SB, Toyoshima H, Wang Z, Pietrobelli A, Heshka S. Race-ethnicity-specific waist circumference cutoffs for identifying cardiovascular disease risk factors. Am J Clin Nutr. (2005) 81:409-15. doi: 10.1093/ajen.81.2.409

10. Yusuf S, Hawken S, Ounpuu S, Bautista L, Franzosi MG, Commerford P, et al. Obesity and the risk of myocardial infarction in 27,000 participants from 52 countries: a case-control study. Lancet. (2005) 366:16409. doi: 10.1016/S0140-6736(05)67663-5

11. Cornier MA, Despres JP, Davis N, Grossniklaus DA, Klein S, Lamarche B, et al. Assessing adiposity: a scientific statement from the American Heart Association. Circulation. (2011) 124:1996-2019. doi: 10.1161/CIR.0b013e318233bc6a

12. Park YS, Kim JS. Obesity phenotype and coronary heart disease risk as estimated by the Framingham risk score. J Korean Med Sci. (2012) 27:2439. doi: $10.3346 / \mathrm{jkms} .2012 .27 .3 .243$

13. Kurian AK, Cardarelli KM. Racial and ethnic differences in cardiovascular disease risk factors: a systematic review. Ethn Dis. (2007) 17:143-52. doi: 10.13016/rsqw-ztls

14. Ribeiro AL, Duncan BB, Brant LC, Lotufo PA, Mill JG, Barreto SM. Cardiovascular health in Brazil: trends and perspectives. Circulation. (2016) 133:422-33. doi: 10.1161/CIRCULATIONAHA.114.008727

15. Almeida RT, Almeida MM, Araujo TM. Abdominal obesity and cardiovascular risk: performance of anthropometric indexes in women. Arq Bras Cardiol. (2009) 92:345-50. doi: 10.1590/S0066-782X20090005 00007

16. do Vale Moreira NC, Montenegro RM, Jr., Meyer HE, Bhowmik B, Mdala I, Siddiquee T, et al. Glycated hemoglobin in the diagnosis of diabetes mellitus in a semi-urban brazilian population. Int J Environ Res Public Health. (2019) 16:3598. doi: 10.3390/ijerph16193598

17. Instituto Brasileiro de Geografia e Estatística. Censo 2010. (2010) Available online at: www.ibge.gov.br/home/estatistica/populacao/censo2010. cooperation. We especially appreciate the logistic and financial support of the Health Department of the city of Pindoretama-CE, particularly the Secretary of Health, Valéria Maria Viana Barbosa. We thank Mr. Phil Riley, a native English speaker, and language expert, for editing the manuscript.

18. R Development Core Team. R: A Language and Environment for Statistical Computing. Vienna, Austria: R Foundation for Statistical Computing (2017).

19. Eng J. Sample size estimation: how many individuals should be studied? Radiology. (2003) 227:309-13. doi: 10.1148/radiol.2272012051

20. Sebold FJ, Schuelter-Trevisol F, Nakashima L, Possamai Della Junior A, Pereira MR, Trevisol DJ. Electrocardiographic changes in adults living in a southern Brazilian city: a population-based study. Rev Port Cardiol. (2015) 34:745-51. doi: 10.1016/j.repce.2015.11.003

21. World Medical Association Declaration of Helsinki. Ethical principles for medical research involving human subjects. JAMA. (2013) 310:21914. doi: 10.1001/jama.2013.281053

22. Friedewald WT, Levy RI, Fredrickson DS. Estimation of the concentration of low-density lipoprotein cholesterol in plasma, without use of the preparative ultracentrifuge. Clin Chem. (1972) 18:499-502. doi: 10.1093/clinchem/18.6.499

23. World Health Organization. Waist Circumference and Waist-Hip Ratio. Report of a WHO Expert Consultation. Geneva, Switzerland: World Health Organization (2008).

24. Ashwell M, Gibson S. Waist-to-height ratio as an indicator of "early health risk': simpler and more predictive than using a" matrix' based on BMI and waist circumference. BMJ Open. (2016) 6:e010159. doi: 10.1136/bmjopen-2015-010159

25. Craig CL, Marshall AL, Sjostrom M, Bauman AE, Booth ML, Ainsworth BE, et al. International physical activity questionnaire: 12-country reliability and validity. Med Sci Sports Exerc. (2003) 35:1381-95. doi: 10.1249/01.MSS.0000078924.6145 3.FB

26. Guidelines for Data Processing and Analysis of the International Physical Activity Questionnaire (IPAQ) - Short and Long Forms (2005). Available online at: https://sites.google.com/site/theipaq/home.

27. World Health Organization. Definition, Diagnosis and Classification of Diabetes Mellitus and its Complications. Report of a WHO Consultation. Geneva 1999.

28. Matthews DR, Hosker JP, Rudenski AS, Naylor BA, Treacher DF, Turner RC. Homeostasis model assessment: insulin resistance and beta-cell function from fasting plasma glucose and insulin concentrations in man. Diabetologia. (1985) 28:412-9. doi: 10.1007/BF00280883

29. World Health Organization. Global Status Report on Noncommunicable Diseases 2014. Geneva, Switzerland: World Health Organization (2014).

30. D’Agostino RB, Vasan RS, Pencina MJ, Wolf PA, Cobain M, Massaro JM, et al. General cardiovascular risk profile for use in primary care - The Framingham Heart Study. Circulation. (2008) 117:743-53. doi: 10.1161/CIRCULATIONAHA.107.699579

31. Chang CL, Marmot MG, Farley TM, Poulter NR. The influence of economic development on the association between education and the risk of acute myocardial infarction and stroke. J Clin Epidemiol. (2002) 55:7417. doi: 10.1016/S0895-4356(02)00413-4

32. Stata Statistical Software. Release 15: College Station, TX. StataCorp. (2017).

33. IBM SPSS. Statistics for Windows. Armonk, NY IBM Corp. (2019).

34. Bazo-Alvarez JC, Quispe R, Peralta F, Poterico JA, Valle GA, Burroughs $\mathrm{M}$, et al. Agreement between cardiovascular disease risk scores in resource-limited settings: evidence from 5 peruvian sites. Crit Pathw Cardiol. (2015) 14:74-80. doi: 10.1097/HPC.00000000000 00045

35. Albertini RA, Ferrer DG, Romagnoli PA, Tinti ME, Amigone JL, Capra R, et al. Association between cardiovascular disease risk scores and subclinical atherosclerosis prevalence in non-elderly adult patients from Argentina. Int J Cardiovasc Imaging. (2017) 33:1521-9. doi: 10.1007/s10554-0171152-9 
36. Cintra F, Bittencourt LR, Santos-Silva R, Andersen M, de Paola A, Poyares D, et al. The association between the Framingham risk score and sleep: a São Pãulo epidemiological sleep study. Sleep Med. (2012) 13:57782. doi: 10.1016/j.sleep.2011.12.016

37. Geldsetzer P, Manne-Goehler J, Theilmann M, Davies JI, Awasthi A, Danaei G, et al. Geographic and sociodemographic variation of cardiovascular disease risk in India: A cross-sectional study of 797,540 adults. PLoS Med. (2018) 15:e1002581. doi: 10.1371/journal.pmed.1002581

38. Montalvan Sanchez EE, Urrutia SA, Rodriguez AA, Duarte G, Murillo A, Rivera R, et al. Cardiovascular risk assessment in the resource limited setting of Western Honduras: an epidemiological perspective. Int J Cardiol Heart Vasc. (2020) 27:100476. doi: 10.1016/j.ijcha.2020.100476

39. Li F, Wang X. Relationship between Framingham risk score and subclinical atherosclerosis in carotid plaques: an in vivo study using multi-contrast MRI. Sci China Life Sci. (2017) 60:23-7. doi: 10.1007/s11427-016-0385-5

40. Instituto de. Pesquisa e Estratégia Econômica do Ceará. Perfil Básico Municipal - Pindoretama. (2012).

41. Ahmed SH, Marjerrison N, Kjollesdal MKR, Stigum H, Htet AS, Bjertness E, et al. Comparison of Cardiovascular Risk Factors among Somalis Living in Norway and Somaliland. Int J Environ Res Public Health. (2019) 16:2353. doi: 10.3390/ijerph16132353

42. Karim R, Hodis HN, Detrano R, Liu CR, Liu CH, Mack WJ. Relation of Framingham risk score to subclinical atherosclerosis evaluated across three arterial sites. Am J Cardiol. (2008) 102:825-30. doi: 10.1016/j.amjcard.2008.05.039

43. Parra FC, Amado RC, Lambertucci JR, Rocha J, Antunes CM, Pena SD. Color and genomic ancestry in Brazilians. Proc Natl Acad Sci U S A. (2003) 100:177-82. doi: 10.1073/pnas.0126614100

44. Reddy KS, Prabhakaran D, Jeemon P, Thankappan KR, Joshi P, Chaturvedi $\mathrm{V}$, et al. Educational status and cardiovascular risk profile in Indians. Proc Natl Acad Sci U S A. (2007) 104:16263-8. doi: 10.1073/pnas.07009 33104

45. Hamdy O, Porramatikul S, Al-Ozairi E. Metabolic obesity: the paradox between visceral and subcutaneous fat. Curr Diabetes Rev. (2006) 2:36773. doi: $10.2174 / 1573399810602040367$
46. Adab P, Pallan M, Whincup PH. Is BMI the best measure of obesity? BMJ. (2018) 360:k1274. doi: 10.1136/bmj.k1274

47. van Dijk SB, Takken T, Prinsen EC, Wittink H. Different anthropometric adiposity measures and their association with cardiovascular disease risk factors: a meta-analysis. Neth Heart J. (2012) 20:208-18. doi: 10.1007/s12471-011-0237-7

48. Ashwell M, Hsieh SD. Six reasons why the waist-to-height ratio is a rapid and effective global indicator for health risks of obesity and how its use could simplify the international public health message on obesity. Int J Food Sci Nutr. (2005) 56:303-7. doi: 10.1080/096374805001 95066

49. D’Agostino RB. Sr., Grundy S, Sullivan LM, Wilson P, Group CHDRP. Validation of the Framingham coronary heart disease prediction scores: results of a multiple ethnic groups investigation. JAMA. (2001) 286:180-7. doi: 10.1001/jama.28 6.2 .180

Conflict of Interest: The authors declare that the research was conducted in the absence of any commercial or financial relationships that could be construed as a potential conflict of interest.

Publisher's Note: All claims expressed in this article are solely those of the authors and do not necessarily represent those of their affiliated organizations, or those of the publisher, the editors and the reviewers. Any product that may be evaluated in this article, or claim that may be made by its manufacturer, is not guaranteed or endorsed by the publisher.

Copyright (c) 2021 Moreira, Mdala, Hussain, Bhowmik, Siddiquee, Fernandes, Montenegro and Meyer. This is an open-access article distributed under the terms of the Creative Commons Attribution License (CC BY). The use, distribution or reproduction in other forums is permitted, provided the original author(s) and the copyright owner(s) are credited and that the original publication in this journal is cited, in accordance with accepted academic practice. No use, distribution or reproduction is permitted which does not comply with these terms. 